\title{
Amelioration of Aluminium Toxicity in Pigeon Pea [Cajanus cajan (L.) Millsp.] Plant by 24-Epibrassinolide
}

\author{
N. Divya Sri, M. Madhan Mohan, K. Mahesh, K. Raghu, S. Seeta Ram Rao* \\ Department of Botany, Osmania University, Hyderabad, India \\ Email: "ssrrao2002@rediffmail.com
}

Received 24 May 2016; accepted 20 August 2016; published 23 August 2016

Copyright $(02016$ by authors and Scientific Research Publishing Inc.

This work is licensed under the Creative Commons Attribution International License (CC BY). http://creativecommons.org/licenses/by/4.0/

c) (i) Open Access

\begin{abstract}
The effect of 24-epibrassinolide on growth of pigeon pea [Cajanus cajan (L.) Millsp.] under aluminium toxicity was studied. 24-EBL reduced the impact of Al stress on plant growth. Particularly 24EBL reduced the inhibitory impact of aluminium toxicity on root growth which was further manifested in overall improvement of vegetative growth. Application of 24-epibrassinolide removed the inhibitory influence of Al nodulation. The growth stimulation in Cajanus plants by 24-EBL under Al stress was associated with elevated levels of chlorophylls, nucleic acids and soluble proteins. 24-Epibrassinolide application enhanced proline content in $\mathrm{Al}^{3+}$ stressed Cajanus plants. Further, the supplementation of 24-epibrassinolide to Al stress treatments increased the activities of antioxidative enzymes viz., catalase [EC 1.11.1.6]; peroxidase [EC 1.11.1.7]; superoxide dismutase [EC 1.15.1.1] and ascorbate peroxidase [EC 1.11.1.11]. Lipid peroxidation induced by Al was found reduced with the supplementation of 24-epibrassinolide. The present studies demonstrated the ameliorating capability of 24-epibrassinolide on the Al induced inhibition of plant growth of $C$. cajan.
\end{abstract}

\section{Keywords}

Aluminium Toxicity, 24-Epibrassinolide, Cajanus cajan, Antioxidative Enzymes

\section{Introduction}

Aluminium is the third most abundant element (after oxygen and silicon) in the earth's crust. The prevalence of toxic $\mathrm{Al}^{3+}$ cations in acidic soils $(\mathrm{pH}<5.0)$ is a major limitation to crop production around the world [1] [2].

\footnotetext{
${ }^{*}$ Corresponding author.
}

How to cite this paper: Divya Sri, N., Madhan Mohan, M., Mahesh, K., Raghu, K. and Seeta Ram Rao, S. (2016) Amelioration of Aluminium Toxicity in Pigeon Pea [Cajanus cajan (L.) Millsp.] Plant by 24-Epibrassinolide. American Journal of Plant Sciences, 7, 1618-1628. http://dx.doi.org/10.4236/ajps.2016.712153 
Excess of aluminium is a major soil constraint to food and biomass production [2]. It is estimated that $40 \%$ of the arable soils of the world are acidic and therefore aluminium poisoning is an important agricultural problem. The initial symptom of Al toxicity is the inhibition of root elongation, which has been proposed to be caused by a number of different mechanisms, including $\mathrm{Al}$ interactions with plasma membrane [3], or the symplast [4]. Aluminium toxicity severely impairs root growth and interferes with water and mineral nutrient uptake. Excess $\mathrm{Al}$ interferes cellular redox equilibrium and boosts the accumulation of reactive oxygen species (ROS), resulting in oxidative damage [5]. Enhanced production of ROS results in oxidative damage to nucleic acids, pigments, proteins and membrane lipids [6]-[8].

Brassinosteroids are new group of phytohormones with significant growth promoting activity [9] [10]. Brassinosteroids are regarded as plant growth regulators with pleiotropic effects as they influence diverse developmental process such as seed germination, plant growth, rhizogenesis, flowering, senescence, and abscission. In addition, brassinosteroids also confer resistance to the plants against abiotic stresses [11] [12].

Pigeon pea [Cajanus cajan (L.) Millsp.] is a tall, fast growing, short-lived perennial shrub that is traditionally cultivated as an annual crop in developing countries. Pigeon pea is one of the major grain pulse crops of the tropics and sub tropics. India is a principal pigeon pea growing country contributing nearly $90 \%$ of the total world production. It is a multipurpose plant as it is extensively eaten as a dal. It is rich in proteins. Pigeon pea is an ideal source of protein and vitamins in human diet, especially in the vegetarian population. It has a long, massive and complex root system which can penetrate to the deeper layers of the soil. Pigeon pea is an important nitrogen fixing pulse crop. The present study aimed to explore the possibility of ameliorating aluminium toxicity in pigeon pea [Cajanus cajan (L.) Millsp.] plant by employing 24-epibrassinolide.

\section{Materials and Methods}

\subsection{Chemicals and Seed Material}

24-epibrassinolide was purchased from CID tech. Research Inc, Mississauga, Ontario, Canada. The seeds of pigeon pea [Cajanus cajan (L.) Millsp.] were procured from National Seed Corporation, Hyderabad, India.

\subsubsection{Chemical Structure of the 24-Epibrassinolide (Figure 1)}

Epibrassinolide (EBL) [24-epibrassinolide, (24R)-brassinolide, (22R, 23R, 24R)-2 $\alpha, 3 \alpha, 22$, 23-tetra-hydroxy24-methyl-B-homo-7-oxa-5a-cholestan-6-one]. $\mathrm{C}_{28} \mathrm{H}_{48} \mathrm{O}_{6}$, MW 480.69.

\subsubsection{Growth Condition and Treatments}

The soil employed for growing plants was analyzed for aluminium by X-ray fluorescence (XRF) spectrometry [13] employing Phillips MagiX PRO, Model PW 2440. The soil was found to contain $13.27 \% \mathrm{Al}_{2} \mathrm{O}_{3}$. Earthen pots were filled with $3 \mathrm{~kg}$ of soil and further supplemented separately with four $\mathrm{Al}$ concentration treatments (as solution) viz.: $0 \mathrm{mg} \cdot \mathrm{kg} \mathrm{soil}^{-1}$, $400 \mathrm{mg} \mathrm{Al} \mathrm{kg} \mathrm{soil}{ }^{-1}, 600 \mathrm{mg} \mathrm{Al} \mathrm{kg} \mathrm{soil}^{-1}$ and $800 \mathrm{mg} \mathrm{Al} \mathrm{kg} \mathrm{soil}^{-1}$ respectively using $\mathrm{Al}_{2}\left(\mathrm{SO}_{4}\right)_{3} \cdot 18 \mathrm{H}_{2} \mathrm{O}$. Seeds were surface sterilized with $0.1 \%$ sodium hypochlorite solution for 10 min and rinsed with double distilled water. The seeds were treated with Rhizobium (IARI, New Delhi). Seeds were sown in earth pots and the pots were kept under natural photoradiation in glass house. On $15^{\text {th }}, 30^{\text {th }}$ and $45^{\text {th }}$ day foliar

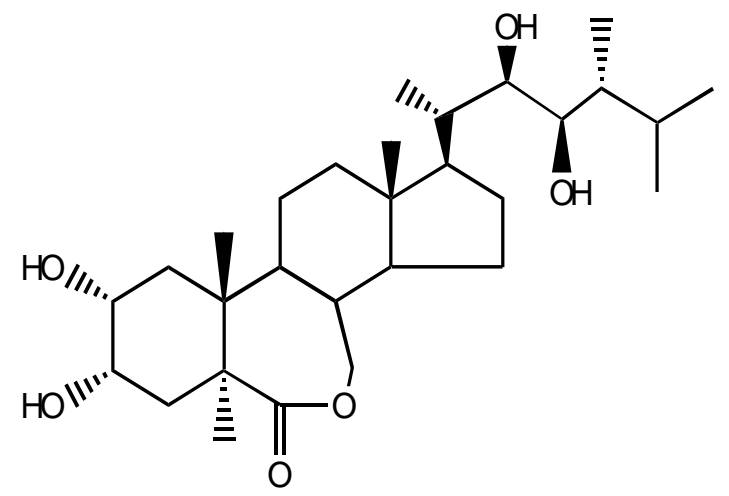

Figure 1. 24-Epibrassinolide. 
spray of 24-epibrassinolide was given at 3 concentration levels $(0.5,1.0$ and $2.0 \mu \mathrm{M})$ separately to plants growing in $\mathrm{Al}$ amended soils viz.: $400 \mathrm{mg} \mathrm{Al} \mathrm{kg} \mathrm{soil}{ }^{-1}, 600 \mathrm{mg} \mathrm{Al} \mathrm{kg} \mathrm{soil}^{-1}$ and $800 \mathrm{mg} \mathrm{Al} \mathrm{kg} \mathrm{soil}{ }^{-1}$ treatments. Based on the results of dose dependent preliminary experiments, three concentrations of brassinosteroids where significant growth promotion was observed (i.e.; 0.5, 1.0 and $2.0 \mu \mathrm{m}$ ) were selected for the experiments. As the responsiveness of the plants to hormones is more during vegetative growth period, brassinosteroids were applied as foliar spray at 15 days intervals started from 15 DAS to 45 DAS. However EBL was not applied to control and $\mathrm{Al}^{+3}$ alone treatments. The pots were supplied with deionized water on alternate days and care was taken to collect the leached out water and the same was again added to the respective pots. Five replicates (pots) with five plants in each pot were maintained for each treatment. After 60-days, the topmost three fully-expanded leaves of each plant were sampled. Each experiment was carried out at least three times.

\subsection{Growth Parameters}

On $60^{\text {th }}$ day, the height of the plant was recorded. After recording the height, the pots were flooded with water and the plants were uprooted carefully from pots and dipped in bucket full of water to remove the adhering soil particles, ensuring safety of roots and then the plants were blotted. Root growth parameters (root length/ square area/volume of the root) were recorded with WinRhizo Root Scanner (XL RHIZO 2012a, Regent Instrument Inc Canada). The number of root nodules per plant was counted for individual treatments. Leaf area of the plant was recorded by distructive method employing leaf area meter (CI-203, CID Inc., Vancouver, Washington-USA). The fresh weights of the roots and shoots were determined separately. The plants were then dried in the oven at $110^{\circ} \mathrm{C}$ for 24 hours and dry weights were recorded.

The leaf material from different treatments was homogenized in $70 \%(\mathrm{v} / \mathrm{v})$ ethyl alcohol and the homogenate was stored in deep freezer $\left(-20^{\circ} \mathrm{C}\right)$ for the estimation of soluble proteins and nucleic acids. However, fresh leaf material was employed for the extraction of photosynthetic pigments, free proline, antioxidant enzymes and MDA content.

\subsection{Chlorophylls}

The chlorophyll pigments were extracted from the leaves and estimated adopting the procedure described by Arnon [14].

\subsubsection{Soluble Proteins}

Soluble proteins in alcohol homogenate (extract in case of enzyme assay) were precipitated by using 20\% (w/v) trichloroacetic acid. The precipitate was dissolved in $5 \mathrm{ml}$ of $1 \%(\mathrm{w} / \mathrm{v})$ sodium hydroxide and was centrifuged at $4000 \mathrm{rpm}$ for $10 \mathrm{~min}$. The supernatant was used for estimation of proteins [15].

\subsubsection{Free Proline}

The amount of proline content was estimated as described by Bates et al. [16]. Plant material (0.5 g) was homogenized with $10 \mathrm{ml}$ of $3 \%(\mathrm{w} / \mathrm{v})$ sulfosalicylic acid and the homogenate was filtered through whatman No. 2 filter paper. The supernatant was taken for proline estimation. To $2 \mathrm{ml}$ of plant extract, $2 \mathrm{ml}$ of acid ninhydrin reagent and $2 \mathrm{ml}$ of glacial acetic acid were added. The test tubes containing above mixture were heated in a boiling water bath for one hour. The reaction was terminated in an ice bath followed by addition of $4 \mathrm{ml}$ of toluene. The contents were shaken vigorously and then allowed to separate into phases. The chromophase containing upper toluene was carefully taken out with the help of a pipette and the absorbance was taken at $520 \mathrm{~nm}$. The amount of proline present was quantified with the help of proline standard graph.

\subsubsection{Nucleic Acids}

DNA and RNA fractions in the ethyl alcohol homogenate were separated [17]. DNA was estimated by employing diphenylamine reagent [18] and RNA was quantified by using Orcinol reagent [19].

\subsubsection{Lipid Peroxidation}

Lipid peroxidation was determined by estimating the malondialdehyde (MDA) content [20]. Leaf material (1.0 g) was homogenized with $3 \mathrm{ml}$ of $0.5 \%$ thiobarbituric acid (TBA) in $20 \%$ trichloroacetic acid (v/v). The homogenate was incubated at $95^{\circ} \mathrm{C}$ for $30 \mathrm{~min}$ and the reaction was stopped in ice. The samples were centrifuged at 
$10,000 \times \mathrm{g}$ for $5 \mathrm{~min}$ at $4^{\circ} \mathrm{C}$ and the absorbance of the resulting supernatant was recorded at $532 \mathrm{~nm}$ and $600 \mathrm{~nm}$. The non-specific absorbance at $600 \mathrm{~nm}$ was subtracted from the $532 \mathrm{~nm}$ absorbance. The absorbance coefficient of MDA was calculated by using the extinction coefficient of $155 \mathrm{mM}^{-1} \cdot \mathrm{cm}^{-1}$.

\subsubsection{Antioxidant Enzymes}

The plant material (200 mg) was homogenized with sodium phosphate buffer at $\mathrm{pH} 7.0$ for CAT, POD, and APX and at $\mathrm{pH} 7.8$ for SOD activities. The supernatant was used to measure the activity of the enzymes.

Catalase (CAT, E.C.1.11.1.6.) activity was determined following the procedure described by Aebi [21]. The reaction mixture consisted of $50 \mathrm{mM}$ phosphate buffer, $0.1 \mathrm{mM} \mathrm{H}_{2} \mathrm{O}_{2}$ and enzyme extract. The rate of $\mathrm{H}_{2} \mathrm{O}_{2}$ decomposition at $240 \mathrm{~nm}$ was measured spectrophotometrically and calculated using a molar extinction coefficient of $45.2 \mathrm{mM}^{-1} \cdot \mathrm{cm}^{-1}$. One unit of catalase activity was assumed as the amount of enzyme that decomposed 1 $\mu \mathrm{mol}$ of $\mathrm{H}_{2} \mathrm{O}_{2}$ per mg of soluble protein per minute at $30^{\circ} \mathrm{C}$.

Peroxidase (POD, E.C.1.11.1.7) activity was assayed by employing the procedure of Kar and Mishra [22]. To $0.5 \mathrm{ml}$ of enzyme extract, $2.5 \mathrm{ml}$ of $0.1 \mathrm{M}$ phosphate buffer ( $\mathrm{pH} 7.0$ ), $1.0 \mathrm{ml}$ of $0.01 \mathrm{M}$ pyrogallol and $1.0 \mathrm{ml}$ of $0.005 \mathrm{M} \mathrm{H}_{2} \mathrm{O}_{2}$ were added. After incubation, the reaction was stopped by adding $1.0 \mathrm{ml}$ of $2.5 \mathrm{~N} \mathrm{H}_{2} \mathrm{SO}_{4}$. The amount of purpurogallin formed was estimated by measuring the absorbance at $420 \mathrm{~nm}$. The enzyme activity was expressed in absorbance units.

Superoxide dismutase (SOD, E.C.1.15.1.1) activity was determined based on inhibition of the photochemical reduction of nitrobluetetrazolium (NBT) [23]. Reaction mixture contained $50 \mathrm{mM}$ sodium phosphate buffer (pH 7.8), $1.5 \mathrm{ml}$ methionine, $1 \mathrm{ml}$ of NBT, $0.75 \mathrm{ml}$ triton-X-100, $2 \mathrm{mM}$ EDTA, $0.1 \mathrm{ml}$ of enzyme extract and $10 \mu \mathrm{L}$ of riboflavin. The reaction mixture was exposed to 15 watt fluorescent tubes and the decrease in the absorbance of the reaction mixture was read at $560 \mathrm{~nm}$. One unit of activity is the amount of protein required to inhibit $50 \%$ initial reduction of NBT under light.

Ascorbate peroxidase (APX, E.C.1.11.1.11) activity was assayed spectrometrically [24]. The reaction mixture contained $50 \mathrm{mM}$ phosphate buffer (pH 7.0), $0.2 \mathrm{mM}$ EDTA, $0.5 \mathrm{mM}$ ascorbic acid, $250 \mathrm{mM} \mathrm{H}_{2} \mathrm{O}_{2}$ and enzyme extract. The activity of APX was measured spectrophotometrically by measuring the rate of ascorbate oxidation at $290 \mathrm{~nm}$ for $1 \mathrm{~min}$. The amount of ascorbate was calculated from the extinction coefficient of $2.6 \mathrm{mM}^{-1} \cdot \mathrm{cm}^{-1}$.

\subsubsection{Statistical Analysis}

The results presented are mean \pm SE of 5 replicates. The data analysis was carried out by one-way analysis of variance (ANOVA) followed by Tukey test of multiple comparisons and $p \leq 0.05$ was considered as significant. In tables comparisons of control Vs all treatments and stress control (400 mg Al/600 mg Al/800 mg Al) Vs respective EBL supplementary concentrations is provided.

\section{Results}

\subsection{Plant Growth}

Aluminium toxicity significantly reduced the growth of pigeon pea plants (Table 1). The growth retardation was found to be correlated with the levels of $\mathrm{Al}$ amendment to the soil. The impact of $\mathrm{Al}$ toxicity was more pronounced on root growth (Table 2). There was severe impairment of root growth in plants growing under elevated $\mathrm{Al}$ content. However, supplementation 24-EBL remarkably reduced the toxic effect on growth. Stress alleviation by 24-EBL was found to be dose dependent manner. $2 \mu \mathrm{M}$ supplementation of 24-EBL to Al challenged plants resulted in restoration of growth almost to the levels of plants growing under unstressed condition. Similarly the decline in foliage growth due to Al toxicity, as reflected in reduced leaf area, was found to be minimized due to 24-EBL application (Table 1). 24-EBL at $2 \mu \mathrm{M}$ concentration accounted for maximum restoration in leaf area in plants growing in soils with added aluminium.

Under Al toxicity, the number of nodules per plant was found reduced (Table 2). However, 24-EBL feeding resulted in improvement in nodulation in plants growing in aluminium rich soil treatments.

\subsection{Chlorophylls}

Reduction in the content of chlorophyll "a", "b" and total chlorophylls have been observed in the $\mathrm{Al}^{3+}$ stressed Cajanus plants when compared to control (Table 3). The reduction in pigment levels in pigeon pea plant was 
Table 1. Effect of 24-EBL on the growth of Cajanus cajan plants grown under $\mathrm{Al}^{3+}$ stress.

\begin{tabular}{|c|c|c|c|c|}
\hline Treatment & Plant height $(\mathrm{cm})$ & Shoot FW (g) & Shoot DW (g) & Leaf Area $\left(\mathrm{cm}^{2}\right)$ \\
\hline Control & $33.5 \pm 1.3$ & $68.6 \pm 12.0$ & $7.4 \pm 0.9$ & $14.1 \pm 0.9$ \\
\hline $\mathrm{Al} 400 \mathbf{~ m g} \cdot \mathbf{k g ~ s o i l}{ }^{-1}$ & $27.9 \pm 1.2$ & $51.3 \pm 9.3$ & $6.3 \pm 0.8$ & $11.2 \pm 0.7$ \\
\hline $\mathrm{Al} 400 \mathrm{mg} \cdot \mathrm{kg}$ soil $^{-1}+0.5 \mu \mathrm{MEBL}$ & $28.4 \pm 1.0$ & $49.4 \pm 6.3$ & $5.9 \pm 0.9^{@}$ & $11.5 \pm 0.6$ \\
\hline $\mathrm{Al} 400 \mathbf{~ m g} \cdot \mathbf{k g ~ s o i l} \mathbf{l}^{-1}+1 \boldsymbol{\mu}$ MEBL & $31.6 \pm 1.5^{*}$ & $58.3 \pm 7.9^{@}$ & $6.3 \pm 0.3^{* @}$ & $12.1 \pm 0.7^{*}$ \\
\hline $\mathrm{Al} 400 \mathbf{~ m g} \cdot \mathbf{k g}$ soil $^{-1}+2 \boldsymbol{\mu M E B L}$ & $34.6 \pm 1.5^{* @}$ & $70.5 \pm 7.1^{* @}$ & $7.1 \pm 0.7^{* @}$ & $13.8 \pm 0.6^{* @}$ \\
\hline $\mathrm{Al} 600 \mathbf{~ m g} \cdot \mathbf{k g}$ soil $^{-1}$ & $28.1 \pm 1.2$ & $47.4 \pm 9.0$ & $5.8 \pm 0.6$ & $11.5 \pm 0.8$ \\
\hline Al $600 \mathbf{~ m g} \cdot \mathbf{k g}$ soil $^{-1}+0.5 \mu \mathrm{MEBL}$ & $28.2 \pm 1.4^{*}$ & $51.1 \pm 10.0^{\dagger}$ & $6.5 \pm 0.5$ & $11.9 \pm 0.8^{\dagger}$ \\
\hline $\mathrm{Al} 600 \mathbf{~ m g} \cdot \mathbf{k g}$ soil $^{-1}+1 \boldsymbol{\mu}$ MEBL & $31.2 \pm 1.2^{*} \dagger$ & $58.4 \pm 8.0^{* \dagger}$ & $6.9 \pm 0.9^{* \dagger}$ & $12.4 \pm 0.9^{* \dagger}$ \\
\hline $\mathrm{Al} 600 \mathbf{~ m g} \cdot \mathbf{k g}$ soil $^{-1}+2 \boldsymbol{\mu M E B L}$ & $31.7 \pm 1.6^{*} \dagger$ & $67.4 \pm 6.9^{* \dagger}$ & $7.2 \pm 0.5^{* \dagger}$ & $14.2 \pm 0.8^{*}$ \\
\hline Al $800 \mathbf{~ m g} \cdot \mathbf{k g}$ soil $^{-1}$ & $25.7 \pm 1.4$ & $37.5 \pm 6.8$ & $3.7 \pm 0.4$ & $09.4 \pm 0.4^{*}$ \\
\hline $\mathrm{Al} 800 \mathrm{mg} \cdot \mathrm{kg} \mathrm{soil}{ }^{-1}+0.5 \mu \mathrm{MEBL}$ & $27.4 \pm 1.3^{\&}$ & $49.5 \pm 11.7^{\&}$ & $6.1 \pm 0.8^{\&}$ & $10.1 \pm 0.3^{*}$ \\
\hline $\mathrm{Al} 800 \mathbf{~ m g} \cdot \mathbf{k g ~ s o i l} \mathbf{l}^{-1}+1 \boldsymbol{\mu}$ MEBL & $31.2 \pm 1.0^{* \&}$ & $56.2 \pm 10.6^{* \&}$ & $6.8 \pm 0.7^{* \&}$ & $11.9 \pm 0.6^{* \&}$ \\
\hline $\mathrm{Al} 800 \mathbf{~ m g} \cdot \mathbf{k g ~ s o i l}{ }^{-1}+2 \boldsymbol{\mu M E B L}$ & $32.1 \pm 0.9^{* \&}$ & $63.4 \pm 5.0^{* \&}$ & $7.3 \pm 0.7^{*}$ & $13.8 \pm 0.7^{* \&}$ \\
\hline
\end{tabular}

The data presented above are Mean \pm S.E. $(n=5)$. Al: Aluminium; EBL $=24$-epibrassinolide. ${ }^{*},{ }^{@},{ }^{\dagger}$ and ${ }^{\&}$ denotes that the mean values are significantly different from unstressed control, $400 \mathrm{mg} \mathrm{Al}, 600 \mathrm{mg} \mathrm{Al}, 800 \mathrm{mg}$ Al controls respectively.

Table 2. Effect of 24-EBL on root growth and nodulation of Cajanus cajan plants grown under $\mathrm{Al}^{3+}$ stress.

\begin{tabular}{|c|c|c|c|c|c|c|}
\hline Treatment & $\begin{array}{l}\text { Root length } \\
\text { (cm) }\end{array}$ & $\begin{array}{l}\text { Root square } \\
\text { area }\left(\mathrm{cm}^{2}\right)\end{array}$ & $\begin{array}{l}\text { Root volume } \\
\left(\mathrm{cm}^{3}\right)\end{array}$ & $\begin{array}{l}\text { Root FW } \\
\text { (mg) }\end{array}$ & $\begin{array}{l}\text { Root DW } \\
\text { (mg) }\end{array}$ & $\begin{array}{c}\text { No. of } \\
\text { root nodules }\end{array}$ \\
\hline Control & $12.7 \pm 0.9$ & $18.6 \pm 1.2$ & $0.39 \pm 0.19$ & $14.1 \pm 1.1$ & $1.51 \pm 0.11$ & $20 \pm 4$ \\
\hline $\mathrm{Al} 400 \mathbf{~ m g} \cdot \mathbf{k g}$ soil $^{-1}$ & $11.5 \pm 0.6$ & $11.5 \pm 1.1$ & $0.29 \pm 0.12$ & $13.6 \pm 1.2$ & $1.42 \pm 0.14$ & $17 \pm 4$ \\
\hline $\mathrm{Al} 400 \mathrm{mg} \cdot \mathbf{k g ~ s o i l}{ }^{-1}+0.5 \mu \mathrm{MEBL}$ & $11.9 \pm 0.8^{@}$ & $12.4 \pm 1.0^{*}$ & $0.35 \pm 0.13^{*}$ & $13.7 \pm 1.1^{@}$ & $1.43 \pm 0.17^{@}$ & $22 \pm 2^{*}$ \\
\hline Al $400 \mathbf{~ m g} \cdot \mathbf{k g}$ soil $^{-1}+1 \mu$ MEBL & $12.2 \pm 0.6^{*} @$ & $13.6 \pm 1.0^{*} @$ & $0.42 \pm 0.15^{*} @$ & $14.1 \pm 1.5^{*} @$ & $1.48 \pm 0.24^{* @}$ & $26 \pm 4^{* @}$ \\
\hline Al $400 \mathbf{~ m g} \cdot \mathbf{k g ~ s o i l}{ }^{-1}+2 \mu$ MEBL & $12.6 \pm 0.5^{*} @$ & $14.6 \pm 1.2^{* @}$ & $0.55 \pm 0.17^{*} @$ & $14.3 \pm 1.6^{*}$ & $1.52 \pm 0.37^{* @}$ & $28 \pm 7^{* @}$ \\
\hline $\mathrm{Al} 600 \mathbf{~ m g} \cdot \mathbf{k g}$ soil $^{-1}$ & $10.9 \pm 0.6$ & $9.60 \pm 1.1$ & $0.23 \pm 0.14^{*}$ & $12.1 \pm 1.0$ & $1.23 \pm 0.10$ & $16 \pm 4$ \\
\hline $\mathrm{Al} 600 \mathrm{mg} \cdot \mathbf{k g ~ s o i l}{ }^{-1}+0.5 \mu \mathrm{MEBL}$ & $10.9 \pm 0.9$ & $10.9 \pm 1.3^{\dagger}$ & $0.32 \pm 0.11^{* \dagger}$ & $12.8 \pm 1.1^{\dagger}$ & $1.28 \pm 0.21^{\dagger}$ & $22 \pm 6^{*+}$ \\
\hline Al $600 \mathbf{~ m g} \cdot \mathbf{k g ~ s o i l}{ }^{-1}+1 \mu$ MEBL & $11.4 \pm 0.5^{* \dagger}$ & $12.5 \pm 1.2^{* \dagger}$ & $0.36 \pm 0.10^{* \dagger}$ & $13.4 \pm 1.5^{* \dagger}$ & $1.37 \pm 0.45^{\dagger}$ & $26 \pm 9^{*}$ \\
\hline Al $600 \mathbf{~ m g} \cdot \mathbf{k g ~ s o i l}{ }^{-1}+2 \boldsymbol{\mu M E B L}$ & $12.6 \pm 0.7^{* \dagger}$ & $14.6 \pm 1.2^{* \dagger}$ & $0.37 \pm 0.14^{\dagger}$ & $13.9 \pm 1.6^{* \dagger}$ & $1.49 \pm 0.68^{*}$ & $30 \pm 4^{*}$ \\
\hline $\mathrm{Al} 800 \mathbf{~ m g} \cdot \mathbf{k g}$ soil $^{-1}$ & $10.3 \pm 0.8$ & $8.61 \pm 1.2$ & $0.20 \pm 0.09$ & $10.8 \pm 0.9$ & $1.04 \pm 0.09$ & $15 \pm 4$ \\
\hline $\mathrm{Al} 800 \mathbf{~ m g} \cdot \mathbf{k g ~ s o i l}{ }^{-1}+0.5 \mu \mathrm{MEBL}$ & $10.7 \pm 0.3^{*}$ & $11.8 \pm 1.2^{*}$ & $0.21 \pm 0.10^{\&}$ & $11.6 \pm 0.9^{* \&}$ & $1.16 \pm 0.65^{\&}$ & $23 \pm 6^{* \&}$ \\
\hline $\mathrm{Al} 800 \mathrm{mg} \cdot \mathbf{k g}$ soil $^{-1}+1 \boldsymbol{\mu M E B L}$ & $12.1 \pm 0.8^{* \&}$ & $13.6 \pm 1.3^{* \&}$ & $0.27 \pm 0.13^{\&}$ & $12.9 \pm 1.5^{* \&}$ & $1.32 \pm 0.81^{\&}$ & $27 \pm 3^{* \&}$ \\
\hline Al $800 \mathbf{~ m g} \cdot \mathbf{k g ~ s o i l}{ }^{-1}+2 \mu$ MEBL & $12.9 \pm 0.7^{* \&}$ & $14.1 \pm 2.1^{* \&}$ & $0.34 \pm 0.12^{* \&}$ & $14.2 \pm 1.9^{*}$ & $1.48 \pm 0.79^{*}$ & $28 \pm 7^{*}$ \\
\hline
\end{tabular}

The data presented above are Mean \pm S.E. $(n=5)$. Al: Aluminium; EBL $=24$-epibrassinolide. ${ }^{*},{ }^{@},{ }^{\dagger}$ and ${ }^{\&}$ denotes that the mean values are significantly different from unstressed control, $400 \mathrm{mg}$ Al, $600 \mathrm{mg} \mathrm{Al}, 800 \mathrm{mg}$ Al controls respectively.

Table 3. Effect of 24-EBL on photosynthetic chlorophyll content in Cajanus plants grown under $\mathrm{Al}^{3+}$ stress.

\begin{tabular}{|c|c|c|c|}
\hline Treatment & Chlorophyll a $\left(\mathrm{mg} \cdot \mathrm{g}^{-1} \mathrm{FW}\right)$ & Chlorophyll b (mg $\left.\cdot \mathrm{g}^{-1} \mathrm{FW}\right)$ & Total chlorophylls \\
\hline Control & $2.002 \pm 0.309$ & $1.241 \pm 0.305$ & $3.243 \pm 0.614$ \\
\hline Al $400 \mathbf{~ m g} \cdot \mathbf{k g}$ soil $^{-1}$ & $1.818 \pm 0.172$ & $1.125 \pm 0.215$ & $2.943 \pm 0.387$ \\
\hline $\mathrm{Al} 400 \mathbf{~ m g} \cdot \mathbf{k g ~ s o i l}{ }^{-1}+0.5 \mu \mathrm{MEBL}$ & $1.959 \pm 0.168^{@}$ & $1.221 \pm 0.134$ & $3.180 \pm 0.302^{@}$ \\
\hline Al $400 \mathbf{~ m g} \cdot \mathbf{k g}$ soil $^{-1}+1 \mu$ MEBL & $2.048 \pm 0.132^{@}$ & $1.343 \pm 0.157^{@}$ & $3.391 \pm 0.289^{* @}$ \\
\hline $\mathrm{Al} 400 \mathbf{~ m g} \cdot \mathbf{k g ~ s o i l}{ }^{-1}+2 \mu \mathrm{MEBL}$ & $2.091 \pm 0.238^{@}$ & $1.505 \pm 0.135^{@}$ & $3.596 \pm 0.373^{* @}$ \\
\hline Al $600 \mathbf{~ m g} \cdot \mathbf{k g}$ soil $^{-1}$ & $1.471 \pm 0.215^{*}$ & $0.743 \pm 0.145^{*}$ & $2.228 \pm 0.360$ \\
\hline $\mathrm{Al} 600 \mathbf{~ m g} \cdot \mathbf{k g ~ s o i l}{ }^{-1}+0.5 \mu$ MEBL & $1.681 \pm 0.201^{* \dagger}$ & $0.842 \pm 0.159^{* \dagger}$ & $2.523 \pm 0.360^{\dagger}$ \\
\hline Al $600 \mathbf{~ m g} \cdot \mathbf{k g}$ soil $^{-1}+1 \boldsymbol{\mu M E B L}$ & $1.991 \pm 0.120^{\dagger}$ & $0.907 \pm 0.186^{* \dagger}$ & $2.898 \pm 0.306^{* \dagger}$ \\
\hline $\mathrm{Al} 600 \mathbf{~ m g} \cdot \mathbf{k g}$ soil $^{-1}+2 \mu \mathrm{MEBL}$ & $2.003 \pm 0.251^{\dagger}$ & $1.138 \pm 0.190^{\dagger}$ & $3.141 \pm 0.441^{* \dagger}$ \\
\hline $\mathrm{Al} 800 \mathbf{~ m g} \cdot \mathbf{k g ~ s o i l}{ }^{-1}$ & $0.959 \pm 0.168^{*}$ & $0.235 \pm 0.147^{*}$ & $1.194 \pm 0.315$ \\
\hline $\mathrm{Al} 800 \mathbf{~ m g} \cdot \mathbf{k g ~ s o i l}{ }^{-1}+0.5 \mu \mathrm{MEBL}$ & $1.087 \pm 0.224^{* \&}$ & $0.493 \pm 0.164^{*}$ & $1.580 \pm 0.388^{\&}$ \\
\hline $\mathrm{Al} 800 \mathbf{~ m g} \cdot \mathbf{k g}$ soil $^{-1}+1 \boldsymbol{\mu}$ MEBL & $1.475 \pm 0.146^{* \&}$ & $0.787 \pm 0.160^{* \&}$ & $2.202 \pm 0.306^{\&}$ \\
\hline $\mathrm{Al} 800 \mathrm{mg} \cdot \mathbf{k g}$ soil $^{-1}+2 \boldsymbol{\mu M E B L}$ & $1.720 \pm 0.234^{* \&}$ & $0.992 \pm 0.100^{\&}$ & $2.712 \pm 0.334^{\&}$ \\
\hline
\end{tabular}

The data presented above are Mean \pm S.E. $(\mathrm{n}=5)$. Al: Aluminium; EBL $=24$-epibrassinolide. ${ }^{*},{ }^{@},{ }^{\dagger}$ and ${ }^{\&}$ denotes that the mean values are significantly different from unstressed control, $400 \mathrm{mg} \mathrm{Al}, 600 \mathrm{mg} \mathrm{Al}, 800 \mathrm{mg}$ Al controls respectively. 
found to be dose dependent. At high aluminium levels the pigment contents were very low. Foliar application of 24-epibrassinolide to $\mathrm{Al}^{3+}$ stressed plants improved the chlorophyll content over the $\mathrm{Al}^{3+}$ alone treatments. $2 \mu \mathrm{M}$ concentration of 24-EBL was found to be most effective in restoring the contents of chlorophyll pigments.

\subsection{Metabolites}

The plants grown in aluminium fortified soils exhibited reduced levels of nucleic acids (Table 4). However foliar application of 24-epibrassinolide restored the nucleic acid levels to the levels found in unstressed control plants.

There was a great decline in leaf protein content in Cajanus plants growing under elevated levels of aluminium (Table 4). Exogenous application of 24-epibrassinolide removed the negative impact of aluminium on protein content.

Compared to the control, free proline levels in Al stressed plants significantly increased (Table 4). Due to supplementation of 24-EBL to Al stressed plants, proline levels were further enhanced. The maximum increase in proline levels was observed in $2 \mu \mathrm{M}$ EBL treated plants growing in high $\mathrm{Al}$ content (800 mg Al kg soil ${ }^{-1}$ ).

\subsection{Antioxidant Enzyme Activities}

Gradual increase in catalase activity in Cajanus plants proportionate to the levels of Al toxicity stress (Table 5). Application of 24-EBL resulted in furtherance of catalase activity in dose dependent manner. Plants treated with $2 \mu \mathrm{M}$ 24-EBL concentration exhibited maximum catalase activity.

The activity of peroxidase enzyme in Cajanus plants was found declined due to $\mathrm{Al}$ toxicity stress (Table 5). The decline in the peroxidase under aluminium stress was found negated by 24-epibrassinolide. $2 \mu \mathrm{M}$ concentrations proved to be more effective in accounting increase in peroxidase activity in all the three levels of added aluminium.

Cajanus plants under $\mathrm{Al}^{3+}$ toxicity exhibited increase in APX activity when compared with control (Table 5). The increase in APX activity was found to be aluminium dose dependent. 24-EBL supplementation to plants under $\mathrm{Al}^{3+}$-stress further enhanced the APX activity at all the three levels of aluminium toxicity stress.

Cajanus plants grown under various levels of Al toxicity showed significant enhancement in the activity of SOD in comparison to unstressed control plants (Table 5). 24-Epibrassinolide supplementation to aluminium plants resulted in furtherance of SOD activity.

\subsection{MDA Content}

Cajanus plants under aluminium toxicity showed higher levels of MDA content indicating lipid peroxidation (Table 6). The accumulation of MDA in cajanus plants was found to be dose dependent. 24-Epibrassinolide ap-

Table 4. Effect of 24-EBL on the content of nucleic acids, soluble proteins and free proline in Cajanus plants grown under $\mathrm{Al}^{3+}$ stress.

\begin{tabular}{|c|c|c|c|c|}
\hline Treatment & $\begin{array}{c}\text { DNA } \\
\left(\mathrm{mg} \cdot \mathrm{g}^{-1} \mathrm{FW}\right)\end{array}$ & $\begin{array}{c}\text { RNA } \\
\left(\mathrm{mg} \cdot \mathrm{g}^{-1} \mathrm{FW}\right)\end{array}$ & $\begin{array}{l}\text { Soluble protein } \\
\left(\mathrm{mg} \cdot \mathrm{g}^{-1} \mathrm{FW}\right)\end{array}$ & $\begin{array}{l}\text { Free proline } \\
\left(\mathrm{mg} \cdot \mathrm{g}^{-1} \mathrm{FW}\right)\end{array}$ \\
\hline Control & $0.541 \pm 0.022$ & $0.839 \pm 0.011$ & $1.68 \pm 0.34$ & $3.22 \pm 0.20$ \\
\hline $\mathrm{Al} 400 \mathbf{~ m g} \cdot \mathbf{k g}$ soil $^{-1}$ & $0.436 \pm 0.015^{*}$ & $0.790 \pm 0.016^{*}$ & $1.32 \pm 0.19^{*}$ & $4.12 \pm 0.19^{*}$ \\
\hline $\mathrm{Al} 400 \mathrm{mg} \cdot \mathbf{k g ~ s o i l}{ }^{-1}+0.5 \mu \mathrm{MEBL}$ & $0.439 \pm 0.014^{*}$ & $0.796 \pm 0.028$ & $1.37 \pm 0.21$ & $4.63 \pm 0.16^{*} @$ \\
\hline Al $400 \mathbf{~ m g} \cdot \mathbf{k g}$ soil $^{-1}+1 \boldsymbol{\mu}$ MEBL & $0.456 \pm 0.024^{*}$ & $0.811 \pm 0.026$ & $1.54 \pm 0.36^{*}$ & $5.13 \pm 0.22^{* @}$ \\
\hline Al $400 \mathbf{~ m g} \cdot \mathbf{k g}$ soil $^{-1}+2 \mu$ MEBL & $0.508 \pm 0.055$ & $0.837 \pm 0.029^{@}$ & $1.67 \pm 0.43$ & $6.54 \pm 0.17^{* @}$ \\
\hline Al $600 \mathbf{~ m g} \cdot \mathbf{k g}$ soil $^{-1}$ & $0.430 \pm 0.032^{*}$ & $0.725 \pm 0.044^{*}$ & $1.14 \pm 0.18^{*}$ & $4.91 \pm 0.16^{*}$ \\
\hline $\mathrm{Al} 600 \mathrm{mg} \cdot \mathbf{k g ~ s o i l}{ }^{-1}+0.5 \mu \mathrm{MEBL}$ & $0.395 \pm 0.028^{* \dagger}$ & $0.731 \pm 0.022^{*}$ & $1.18 \pm 0.16$ & $5.20 \pm 0.26^{* \dagger}$ \\
\hline Al $600 \mathbf{~ m g} \cdot \mathbf{k g}$ soil $^{-1}+1 \boldsymbol{\mu}$ MEBL & $0.412 \pm 0.029^{* \dagger}$ & $0.769 \pm 0.025^{* \dagger}$ & $1.26 \pm 0.32^{*}$ & $5.68 \pm 0.12^{* \dagger}$ \\
\hline Al $600 \mathbf{~ m g} \cdot \mathbf{k g}$ soil $^{-1}+2 \mu$ MEBL & $0.447 \pm 0.031^{* \dagger}$ & $0.784 \pm 0.027^{*}$ & $1.34 \pm 0.43^{*}$ & $6.88 \pm 0.13^{* \dagger}$ \\
\hline Al $800 \mathbf{~ m g} \cdot \mathbf{k g}$ soil $^{-1}$ & $0.384 \pm 0.025^{*}$ & $0.620 \pm 0.052^{*}$ & $1.06 \pm 0.12^{*}$ & $6.20 \pm 0.20^{*}$ \\
\hline $\mathrm{Al} 800 \mathrm{mg} \cdot \mathbf{k g ~ s o i l}{ }^{-1}+0.5 \mu \mathrm{MEBL}$ & $0.394 \pm 0.027^{*}$ & $0.655 \pm 0.032^{*}$ & $1.13 \pm 0.12$ & $6.13 \pm 0.28^{*}$ \\
\hline Al $800 \mathbf{~ m g} \cdot \mathbf{k g}$ soil $^{-1}+1 \boldsymbol{\mu}$ MEBL & $0.409 \pm 0.021^{* \&}$ & $0.732 \pm 0.039^{* \&}$ & $1.28 \pm 0.23^{*}$ & $8.74 \pm 0.32^{* \&}$ \\
\hline Al $800 \mathbf{~ m g} \cdot \mathbf{k g}$ soil $^{-1}+2 \mu$ MEBL & $0.443 \pm 0.030^{* \&}$ & $0.740 \pm 0.024^{* \&}$ & $1.32 \pm 0.37^{*}$ & $10.2 \pm 0.22^{* \&}$ \\
\hline
\end{tabular}

The data presented above are Mean \pm S.E. $(n=5)$. Al: Aluminium; EBL $=24$-epibrassinolide. ${ }^{*},^{\varrho},{ }^{\dagger}$ and $^{*}$ denotes that the mean values are significantly different from unstressed control, $400 \mathrm{mg} \mathrm{Al,} 600 \mathrm{mg} \mathrm{Al}, 800 \mathrm{mg} \mathrm{Al}$ controls respectively. 
Table 5. Effect of 24-EBL on the activities of antioxidative enzymes in Cajanus plants under $\mathrm{Al}^{3+}$ stress and stress free conditions.

\begin{tabular}{|c|c|c|c|c|}
\hline Treatments & $\begin{array}{c}\text { CAT } \\
\left(\mathrm{U} \cdot \mathrm{mg}^{-1} \text { protein } \text { min }^{-1}\right)\end{array}$ & $\begin{array}{c}\text { POD } \\
\left(\mathrm{U} \cdot \mathrm{mg}^{-1} \text { protein } \mathrm{min}^{-1}\right)\end{array}$ & 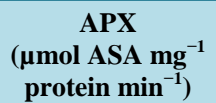 & $\begin{array}{c}\text { SOD } \\
\left(\mathrm{U} \cdot \mathrm{mg}^{-1} \text { protein } \mathrm{min}^{-1}\right)\end{array}$ \\
\hline Control & $1.81 \pm 0.60$ & $0.048 \pm 0.017$ & $4.81 \pm 0.96$ & $1.45 \pm 0.21$ \\
\hline $\mathrm{Al} 400 \mathbf{~ m g} \cdot \mathbf{k g}$ soil $^{-1}$ & $1.88 \pm 0.31$ & $0.041 \pm 0.006$ & $4.93 \pm 0.99$ & $1.61 \pm 0.33$ \\
\hline $\mathrm{Al} 400 \mathrm{mg} \cdot \mathbf{k g ~ s o i l} \mathrm{l}^{-1}+0.5 \mu \mathrm{MEBL}$ & $1.92 \pm 0.52$ & $0.043 \pm 0.017$ & $4.98 \pm 0.93$ & $1.85 \pm 0.51$ \\
\hline Al $400 \mathbf{~ m g} \cdot \mathbf{k g ~ s o i l}{ }^{-1}+1 \boldsymbol{\mu M E B L}$ & $1.99 \pm 0.64^{*}$ & $0.046 \pm 0.008$ & $5.12 \pm 1.04$ & $1.92 \pm 0.62$ \\
\hline Al $400 \mathbf{~ m g} \cdot \mathbf{k g}$ soil $^{-1}+2 \boldsymbol{\mu M E B L}$ & $2.13 \pm 0.69^{*}$ & $0.048 \pm 0.016$ & $5.45 \pm 1.08^{* @}$ & $2.08 \pm 0.56^{@}$ \\
\hline $\mathrm{Al} 600 \mathbf{~ m g} \cdot \mathbf{k g}$ soil $^{-1}$ & $2.09 \pm 0.28$ & $0.038 \pm 0.012$ & $5.18 \pm 1.02$ & $1.88 \pm 0.41$ \\
\hline $\mathrm{Al} 600 \mathbf{~ m g} \cdot \mathbf{k g ~ s o i l} \mathrm{l}^{-1}+0.5 \mu \mathrm{MEBL}$ & $2.32 \pm 0.53^{*}$ & $0.039 \pm 0.011$ & $5.37 \pm 1.07$ & $1.97 \pm 0.71$ \\
\hline $\mathrm{Al} 600 \mathbf{~ m g} \cdot \mathbf{k g}$ soil $^{-1}+1 \boldsymbol{\mu M E B L}$ & $2.69 \pm 0.62^{*}$ & $0.041 \pm 0.009$ & $5.46 \pm 1.08$ & $2.09 \pm 0.63$ \\
\hline Al $600 \mathbf{~ m g} \cdot \mathbf{k g}$ soil $^{-1}+2 \boldsymbol{\mu M E B L}$ & $2.58 \pm 0.68$ & $0.043 \pm 0.012$ & $5.49 \pm 1.03$ & $2.51 \pm 0.78$ \\
\hline $\mathrm{Al} 800 \mathbf{~ m g} \cdot \mathbf{k g}$ soil $^{-1}$ & $2.41 \pm 0.21$ & $0.032 \pm 0.011$ & $5.44 \pm 1.00$ & $1.93 \pm 0.46$ \\
\hline $\mathrm{Al} 800 \mathbf{~ m g} \cdot \mathbf{k g ~ s o i l}{ }^{-1}+0.5 \mu \mathrm{MEBL}$ & $2.48 \pm 0.41^{*}$ & $0.035 \pm 0.007$ & $5.59 \pm 1.01^{*}$ & $1.95 \pm 0.81$ \\
\hline $\mathrm{Al} 800 \mathbf{~ m g} \cdot \mathbf{k g}$ soil $^{-1}+1 \boldsymbol{\mu M E B L}$ & $2.83 \pm 0.49^{*}$ & $0.039 \pm 0.009$ & $5.82 \pm 1.04^{*}$ & $2.41 \pm 0.94$ \\
\hline Al $800 \mathbf{~ m g} \cdot \mathbf{k g}$ soil $^{-1}+2 \mu \mathrm{MEBL}$ & $3.01 \pm 0.86^{*}$ & $0.045 \pm 0.014^{\&}$ & $6.01 \pm 1.17^{*}$ & $2.99 \pm 0.98^{*}$ \\
\hline
\end{tabular}

The data presented above are Mean \pm S.E. $(n=5)$. Al: Aluminium; EBL $=24$-epibrassinolide; ${ }^{*}$ @ $^{\dagger}{ }^{\dagger}$ and ${ }^{\&}$ denotes that the mean values are significantly different from unstressed control, $400 \mathrm{mg} \mathrm{Al,} 600 \mathrm{mg} \mathrm{Al}, 800 \mathrm{mg} \mathrm{Al}$ controls respectively.

Table 6. Effect of brassinosteroids on lipid peroxidation in Cajanus plants under $\mathrm{Al}^{3+}$ stress.

\begin{tabular}{|c|c|}
\hline Treatments & MDA content $\left(\mu \mathrm{mol} \cdot \mathrm{g}^{-1} \mathrm{FW}\right)$ \\
\hline Control & $36.81 \pm 2.43$ \\
\hline $\mathrm{Al} 400 \mathbf{~ m g} \cdot \mathbf{k g}$ soil $^{-1}$ & $45.13 \pm 3.87^{*}$ \\
\hline $\mathrm{Al} 400 \mathrm{mg} \cdot \mathbf{k g ~ s o i l}{ }^{-1}+0.5 \boldsymbol{\mu M E B L}$ & $43.25 \pm 3.34^{* @}$ \\
\hline $\mathrm{Al} 400 \mathbf{~ m g} \cdot \mathbf{k g ~ s o i l}{ }^{-1}+1 \boldsymbol{\mu M E B L}$ & $42.08 \pm 3.76^{* @}$ \\
\hline $\mathrm{Al} 400 \mathbf{~ m g} \cdot \mathbf{k g}$ soil $^{-1}+2 \boldsymbol{\mu M E B L}$ & $39.01 \pm 3.12^{*} @$ \\
\hline $\mathrm{Al} 600 \mathbf{~ m g} \cdot \mathbf{k g}$ soil $^{-1}$ & $47.05 \pm 3.98^{*}$ \\
\hline Al $600 \mathrm{mg} \cdot \mathbf{k g ~ s o i l}{ }^{-1}+0.5 \mu \mathrm{MEBL}$ & $44.06 \pm 3.81^{* \dagger}$ \\
\hline $\mathrm{Al} 600 \mathbf{~ m g} \cdot \mathbf{k g ~ s o i l}{ }^{-1}+1 \boldsymbol{\mu} \mathbf{M E B L}$ & $40.01 \pm 3.59^{* \dagger}$ \\
\hline Al $600 \mathbf{~ m g} \cdot \mathbf{k g}$ soil $^{-1}+2 \mu$ MEBL & $39.88 \pm 3.61^{* \dagger}$ \\
\hline Al $800 \mathrm{mg} \cdot \mathbf{k g ~ s o i l}{ }^{-1}$ & $48.01 \pm 3.82^{*}$ \\
\hline $\mathrm{Al} 800 \mathrm{mg} \cdot \mathbf{k g ~ s o i l}{ }^{-1}+0.5 \mu \mathrm{MEBL}$ & $47.06 \pm 3.64^{*}$ \\
\hline Al $800 \mathbf{~ m g} \cdot \mathbf{k g ~ s o i l}{ }^{-1}+1 \boldsymbol{\mu M E B L}$ & $44.17 \pm 3.91^{* \&}$ \\
\hline Al $800 \mathrm{mg} \cdot \mathbf{k g}$ soil $^{-1}+2 \boldsymbol{\mu M E B L}$ & $41.02 \pm 3.24^{* \&}$ \\
\hline
\end{tabular}

The data presented above are Mean \pm S.E. $(n=5)$. Al: Aluminium; EBL $=24$-epibrassinolide; ${ }^{*},{ }^{\varrho},{ }^{\dagger}$ and ${ }^{\&}$ denotes that the mean values are significantly different from unstressed control, $400 \mathrm{mg} \mathrm{Al}, 600 \mathrm{mg} \mathrm{Al}, 800 \mathrm{mg} \mathrm{Al}$ controls respectively.

plication set aside the toxic effect of aluminium as indicated by lower MDA content reflecting reduced lipid peroxidation (Table 6).

\section{Discussion}

Excess $\mathrm{Al}$ in the growth medium resulted in substantial reduction in growth as reflected in all the vegetative parameters recorded. Moreover, Al toxicity impact was found to be much more severe on the root growth. It has been suggested that the effect of aluminium is initially in root growth inhibition, resulting in lesser exploration of bulk soil leading to reduced uptake of water and nutrients [25]. 24-EBL feeding to plants growing under Al toxicity stress resulted in alleviation of toxicity impact as reflected in improvement of growth. One of the important observation made in the study is the ameliorative capabilities of 24-EBL on Al induced inhibition of root growth. The improvement of root growth as conferred by brassinosteroids further translated into stimulation of overall growth of plants even under $\mathrm{Al}$ toxicity stress. 
Nodulation was decreased in pigeon pea plants grown in Al challenged substratum. However, the application of 24-EBL reduced the impact of $\mathrm{Al}$ on nodulation. It was reported that Rhizobium multiplication and nodule formation were more susceptible aspect of symbiotic relationship to excess Al [26]. Further it has been demonstrated the positive impact of brassinosteroids on nodulation in Arachis hypogaea [27]. Similarly the beneficial effect of brassinosteroids on nodulation of soybean was also reported [28]. The result of the present study clearly indicated the ameliorative impact of the brassinosteroids on nodulation under aluminium toxicity stress.

In pigeon pea plants, Al toxicity resulted in steep decline in the content of chlorophylls. Metal stress is known to interfere with chlorophyll synthesis and also to induce chlorophyll degradation [29]. The treatment of Cajanus plants with 24-EBL considerably increased the photosynthetic pigments and counteracted the damaging effect of $\mathrm{Al}^{+3}$ stress. Brassinosteroids could prevent loss of photosynthetic pigments, either by activating specific genes responsible for chlorophyll synthesis or by reducing the chlorophyll degradation [30]. BRs significantly increased the levels of photosynthetic pigments in maize under salinity stress [31], in tomato under Cd stress [32] and in radish under $\mathrm{Zn}$ stress [33].

Al toxicity resulted in lowered nucleic acid levels in Cajanus plants. However supplementation of 24-EBL to Al stressed plants caused an improvement in nucleic acid content. BRs application markedly increased the DNA and RNA content in maize plants under salinity stress [34]. The ameliorative influence of BRs on salinity stress induced growth inhibition in rice plants was linked to elevated levels of nucleic acids [35].

Cajanus plants subjected to Al toxicity exhibited enhanced levels of free proline. Exogenous application of 24-EBL caused further increase in proline content in cajanus plants growing under excess Al levels. To increase plant tolerance to abiotic stresses plants may accumulate compounds of low molecular mass such as proline. Proline may differently affect stress tolerance, increase the activity of many enzymes and stabilize protein integrity. Proline contributes to maintenance of the redox balance, can regulate development, and is a component of metabolic signaling networks controlling mitochondrial functions, stress relief and development [36]. Proline accumulates in response to abiotic stress and scavenge ROS and is considered as important non enzymatic antioxidant [37] [38]. Brassinosteroids enhanced the levels of free proline content in chick pea under cadmium stress [39]. The alleviation of zinc toxicity in radish by BRs was attributed to enhanced free proline content [40].

\section{Antioxidant Enzyme Activities}

Plants have developed a complex antioxidative system to mitigate and repair oxidative damage. The synchronous modulation of enzymatic and non-enzymatic antioxidant defense mechanisms plays a key role in the ROS detoxification [41] [42]. SOD constitutes the first line of defense against ROS in plants and this enzyme catalyzes the dismutation of $\mathrm{O}_{2}^{-}$to $\mathrm{H}_{2} \mathrm{O}_{2}$ and $\mathrm{O}_{2}$ [43]. In the present study, a significant increase in SOD activity was observed in $\mathrm{Al}^{3+}$ stressed cajanus plants, suggesting its role in removing $\mathrm{O}_{2}^{-}$. Exogenous application of 24EBL further enhanced the SOD activity in $\mathrm{Al}^{3+}$ stressed plants reflecting the enhanced $\mathrm{O}_{2}^{-}$scavenging there by blocking $\mathrm{O}_{2}^{-}$driven cell damage. The decomposition of $\mathrm{O}_{2}^{-}$always accompanied by production of $\mathrm{H}_{2} \mathrm{O}_{2}$ which diffuses across the plasma membrane and is toxic as it acts both as an antioxidant as well as reductant [41]. Subsequently further break down of $\mathrm{H}_{2} \mathrm{O}_{2}$ to $\mathrm{H}_{2} \mathrm{O}$ and $\mathrm{O}_{2}$ is achieved by CAT and peroxidases [44]. The results of present investigation showed that, CAT, APX increased but POD decreased in Al stressed cajanus plants. However, supplementation of EBL to $\mathrm{Al}^{+3}$ stressed plants increased the activities of the all enzymes (CAT, POD and APX). The BRs modulated increase in the activities of antioxidative enzymes, such as SOD, POD, CAT and APX, clearly indicating a protective role of EBL against the Al induced oxidative damage.

In Cajanus plants, aluminium toxicity in dose dependent manner increased the accumulation of MDA content implying enhanced membrane peroxidation. EBL feeding reduced the MDA content in all the stress treatments indicating the reduction in lipid peroxidation thus the results clearly demonstrating the counteracting influence of EBL on Al toxicity impact. EBL application resulted in lowered MDA content in Zea mays leaves under Mn toxicity [45]. Similarly homobrassinolide application caused decrease in MDA content in radish challenged with Cr toxicity [46]. The suppression of Chlorella vulgaris growth by heavy metals (Cd and $\mathrm{Pb}$ ) was restored by reducing MDA accumulation upon exogenous BR supplementation [47]. Exogenous application of 28-homobrassinolide markedly decreased MDA levels in radish seedlings under zinc stress [48]. The decrease in MDA accumulation in EBL treated plants growing under elevated Al levels was found strongly correlated with increased activity of the antioxidant enzymes such as SOD, CAT, POD, APX and production of free proline, a non enzymatic antioxidant indicating the capability of 24-EBL in mitigating the oxidative stress generated due to alumi- 
nium toxicity.

\section{Conclusion}

Foliar application of 24-epibrassinolide markedly negated the impact of aluminium toxicity stress in Cajanus plants. The impact of 24-epibrassinolide particularly was spectacular on root growth. Application of 24-EBL improved the contents of chlorophylls and soluble protein content. EBL application reduced the impact of Al toxicity on membrane peroxidation as evidenced by lowered MDA content. Foliar application of 24-epibrassinolide significantly increased the activities of antioxidative enzymes (CAT, POD, APX and SOD) and proline, (an important non enzymatic antioxidant) in aluminium stressed Cajanus plants. The result of the present investigation clearly demonstrated the ameliorative effect of EBL on Al toxicity imposed growth inhibition.

\section{Acknowledgements}

The financial assistance from University Grants Commission, New Delhi, India is gratefully acknowledged. The authors acknowledge the help of Dr. Krishnan, Environmental Geochemistry Group, National Geophysical Research Institute (NGRI) Hyderabad, India for the analysis of the soil for aluminium content.

\section{References}

[1] Kochian, L.V., Pineros, M.A. and Hoekenga, O.A. (2005) The Physiology, Genetics and Molecular Biology of Plant Aluminum Resistance and Toxicity. Plant and Soil, 274, 175-195. http://dx.doi.org/10.1007/s11104-004-1158-7

[2] Vitorello, V.A., Capaldi, F.R.C. and Stefanuto, V.A. (2005) Recent Advances in Aluminum Toxicity and Resistance in Higher Plants. Brazilian Journal of Plant Physiology, 17, 129-143. http://dx.doi.org/10.1590/S1677-04202005000100011

[3] Piňeros, M.A. and Kochian, L.V. (2001) A Patch-Clamp Study on the Physiology of Aluminum Toxicity and Aluminum Tolerance in Maize. Identification and Characterization of $\mathrm{Al}^{+3}$ Induced Anion Channels. Plant Physiology, 125, 292-305. http://dx.doi.org/10.1104/pp.125.1.292

[4] Kochian, L.V. (1995) Cellular Mechanisms of Aluminium Toxicity and Resistance in Plants. Annual Review of Plant Physiology and Plant Molecular Biology, 46, 237-260. http://dx.doi.org/10.1146/annurev.pp.46.060195.001321

[5] He, H., He, L. and Gu, M. (2014) Role of MicroRNAs in Aluminium Stress in Plants. Plant Cell Reports, 33, 831-836. http://dx.doi.org/10.1007/s00299-014-1565-z

[6] Rao, K.V.M. and Sresty, T.S. (2000) Antioxidative Parameters in the Seedlings of Pigeon Pea (Cajanuscajan (L.) Millsp.) in Response to Zn and Ni Stresses. Plant Science, 157, 113-128. http://dx.doi.org/10.1016/S0168-9452(00)00273-9

[7] Tewari, R.K., Kumar, P. and Sharma, P.N. (2008) Morphology and Physiology of Zinc-Stressed Mulberry Plants. Journal of Plant Nutrition and Soil Science, 171, 286-294. http://dx.doi.org/10.1002/jpln.200700222

[8] Morina, F., Jovanovicb, L., Mojovicc, M., Vidovica, M., Pankovicd, D. and Jovanovic, S.V. (2010) Zinc Induced Oxidative Stress in Verbascum thapsus Is Caused by an Accumulation of Reactive Oxygen Species and Quinhydrone in the Cell Wall. Physiologia Plantarum, 140, 209-224.

[9] Rao, S.S.R., Vardhini, B.V., Sujatha, E. and Anuradha, S. (2002) Brassinosteroids-New Class of Phytohormones. Current Science, 82, 1239-1245.

[10] Bajguz, A. (2007) Metabolism of Brassinosteroids in Plants. Plant Physiology and Biochemistry, 45, 95-107. http://dx.doi.org/10.1016/j.plaphy.2007.01.002

[11] Sasse, J.M. (2003) Physiological Actions of Brassinosteroids: An Update. Journal of Plant Growth Regulation, 22, 276-288. http://dx.doi.org/10.1007/s00344-003-0062-3

[12] Vardhini, B.V., Anuradha, S. and Rao, S.S.R. (2006) Brassinosteroids-New Class of Plant Hormone with Potential to Improve Crop Productivity. Indian Journal of Plant Physiology, 11, 1-12.

[13] Krishna, A.K., Murthy, N.N. and Govil, P.K. (2007) Multielement Analysis of Soils by Wavelength-Dispersive X-Ray Fluorescence Spectrometry. Atomic Spectroscopy, 28, 202-214.

[14] Arnon, D.I. (1949) Copper Enzymes in Isolated Chloroplast Poly Phenol Oxidase in Beta vulgaris. Plant Physiology, 24, 1-15. http://dx.doi.org/10.1104/pp.24.1.1

[15] Lowry, O.H., Rosenbrough, N.J., Farr, A.L. and Randall, R.J. (1951) Protein Measurement with Folin-Phenol Reagent. Journal of Biological Chemistry, 193, 265-275.

[16] Bates, L., Waldren, R.P. and Teare, I.D. (1973) Rapid Determination of Free Proline for Water-Stress Studies. Plant 
and Soil, 39, 205-207. http://dx.doi.org/10.1007/BF00018060

[17] Ogur, M. and Rosen, G. (1950) The Extraction and Estimation of Deoxypentose Nucleic Acid and Pentose Nucleic Acids. Archives of Biochemistry and Biophysics, 25, 262-276.

[18] Burton, K. (1968) Determination of DNA Concentration with Diphenyl Amine. In: Grossman, L. and Meidave, L., Eds., Methods in Enzymology, Academic Press, New York, 163-166.

[19] Schneider, W.C. (1957) Determination of Nucleic Acids in Tissues by Pentose Analysis. In: Colowick, S.P. and Kaplan, W.O., Eds., Methods in Enzymology, Academic Press, New York, 680-684. http://dx.doi.org/10.1016/s0076-6879(57)03442-4

[20] Heath, R.L. and Packer, L. (1968) Photoperoxidation in Isolated Chloroplasts Kinetics and Stoichiometry of Fatty Acid Peroxidation. Archives of Biochemistry and Biophysics, 12, 189-198. http://dx.doi.org/10.1016/0003-9861(68)90654-1

[21] Aebi, H. (1974) Catalase. In: Bergmeyer, H.U., Ed., Methods of Enzymatic Analysis, Verlag Chemie/Academic Press Inc., Weinheim/NewYork, 673-680. http://dx.doi.org/10.1016/b978-0-12-091302-2.50032-3

[22] Kar, M. and Mishra, D. (1976) Catalase, Peroxidase and Polyphenol Oxidase Activities during Rice Leaf Senescence. Plant Physiology, 57, 315-319. http://dx.doi.org/10.1104/pp.57.2.315

[23] Beauchamp, C. and Fridovich, I. (1971) Superoxide Dismutase Improved Assay and an Assay Applicable to Acrylamide Gels. Analytical Biochemistry, 44, 276-287. http://dx.doi.org/10.1016/0003-2697(71)90370-8

[24] Nakano, Y. and Asada, K. (1981) Hydrogen Peroxide Is Scavenged by Ascorbate Specific Peroxidase in Spinach Chloroplasts. Plant and Cell Physiology, 22, 867-880.

[25] Silva, D.M. and Sodek, L. (1997) Effect of Aluminum on Soybean Nodulation and Nodule Activity in a Vertical SplitRoot System. Journal of Plant Nutrition, 20, 963-974. http://dx.doi.org/10.1080/01904169709365309

[26] Wood, M., Cooper, J.E. and Holding, A.J. (1984) Soil Acidity Factor and Nodulation of Trifolium repens. Plant and Soil, 78, 367-379. http://dx.doi.org/10.1007/BF02450370

[27] Vardhini, B.V. and Rao, S.S.R. (1999) Effect of Brassinosteroids on Nodulation and Nitrogenase Activity in Groundnut (Arachis hypogaea L.). Plant Growth Regulation, 28, 165-167. http://dx.doi.org/10.1023/A:1006227417688

[28] Thakur, A.K. and Singh, K.J. (2014) Seed Emergence Is More Appropriate Criterion for Testing Cadmium Toxicity in Soybean. International Journal of Food, Agriculture and Veterinary Sciences, 4, 129-134.

[29] Van Assche, F. and Clijsters, H. (1990) Effects of Metals on Enzyme Activity in Plants. Plant, Cell and Environment, 13, 195-206. http://dx.doi.org/10.1111/j.1365-3040.1990.tb01304.x

[30] Hayat, S., Yadav, S., Wani, A.S., Irfan, M. and Ahamad, A. (2011) Comparative Effect of 28-Homobrassinolide and 24-Epibrassinolide on the Growth, Carbonic Anhydrase and Photosynthetic Efficiency of Lycopersicon esculentum. Photosynthetica, 49, 397-404. http://dx.doi.org/10.1007/s11099-011-0051-x

[31] Agami, R.R. (2013) Alleviating the Adverse Effects of NaCl Stress in Maize Seedlings by Pre-Treating with Salicylic Acid and 24-Epibrassinolide. South African Journal of Botany, 88, 171-177. http://dx.doi.org/10.1016/j.sajb.2013.07.019

[32] Hasan, S.Y., Hayat, S. and Ahamad, A. (2011) Brassinosteroids Protect Photosynthetic Machinery against the Cadmium Induced Oxidative Stress in Two Tomato Cultivars. Chemosphere, 84, 1446-1451. http://dx.doi.org/10.1016/j.chemosphere.2011.04.047

[33] Ramakrishna, B. and Rao, S.S.R. (2015) Foliar Application of Brassinosteroids Alleviates Adverse Effects of Zinc Toxicity in Radish (Raphanus sativus L.) Plants. Protoplasma, 252, 665-677. http://dx.doi.org/10.1007/s00709-014-0714-0

[34] El-Khallal, S.M., Hathout, T.A., Ashour, A.A. and Kerrit, A.A. (2004) Brassinolide and Salicylic Acid Induced Growth, Biochemical Activities and Productivity of Maize Plants Grown under Salt Stress. Research Journal of Agricultural and Biological Sciences, 5, 380-390.

[35] Anuradha, S. and Rao, S.S.R. (2003) Application of Brassinosteroids to Rice Seeds (Oryza sativa) Reduced the Impact of Salt Stress on Growth, Prevented Photosynthetic Pigment Loss and Increased Nitrate Reductase Activity. Plant Growth Regulation, 40, 29-32. http://dx.doi.org/10.1023/A:1023080720374

[36] Lipiec, J., Doussan, C., Nosalewicz, A. and Kondracka, K. (2013) Effect of Drought and Heat Stresses on Plant Growth and Yield: A Review. International Agrophysics, 27, 463-477. http://dx.doi.org/10.2478/intag-2013-0017

[37] Michalak, A. (2006) Phenolic Compounds and Their Antioxidant Activity in Plants Growing under Heavy Metal Stress. Polish Journal of Environmental Studies, 15, 523-530.

[38] Ashraf, M. and Foolad, M.R. (2007) Roles of Glycine Betaine and Proline in Improving Plant Abiotic Stress Resistance. Environmental and Experimental Botany, 59, 206-216. http://dx.doi.org/10.1016/j.envexpbot.2005.12.006

[39] Hasan, S.A., Hayat, S., Ali, B. and Ahmad, A. (2008) 28-Homobrassinolide Protects Chickpea (Cicer arietinum) from Cadmium Toxicity by Stimulating Antioxidants. Environmental Pollution, 151, 60-66. 
http://dx.doi.org/10.1016/j.envpol.2007.03.006

[40] Ramakrishna, B. and Rao, S.S.R. (2012) 24-Epibrassinolide Alleviated Zinc-Induced Oxidative Stress in Radish (Raphanus sativus L.) Seedlings by Enhancing Antioxidative System. Plant Growth Regulation, 68, 249-259. http://dx.doi.org/10.1007/s10725-012-9713-3

[41] Apel, K. and Hirt, H. (2004) Reactive Oxygen Species: Metabolism, Oxidative Stress, and Signal Transduction. Annual Review of Plant Biology, 55, 373-399. http://dx.doi.org/10.1146/annurev.arplant.55.031903.141701

[42] Gratao, P.L., Polle, A., Lea, P.J. and Azevedo, R.A. (2005) Making the Life of Heavy Metal Stressed Plants a Little Easier. Functional Plant Biology, 32, 481-494. http://dx.doi.org/10.1071/FP05016

[43] Alscher, R.G., Erturk, N. and Heath, L.S. (2002) Role of Superoxide Dismutases (SODs) in Controlling Oxidative Stress in Plants. Journal of Experimental Botany, 53, 1331-1341. http://dx.doi.org/10.1093/jexbot/53.372.1331

[44] Anjum, N.A., Umar, S. and Chan, M.T. (2010) Ascorbate-Glutathione Pathway and Stress Tolerance in Plants. Springer, Dordrecht. http://dx.doi.org/10.1007/978-90-481-9404-9

[45] Wang, C., Zhang, S.H., Wang, P.F., Hou, J., Zhang, W.J., Li, W. and Lin, Z.P. (2009) The Effect of Excess Zn on Mineral Nutrition and Antioxidative Response in Rapeseed Seedlings. Chemosphere, 75, 1468-1476. http://dx.doi.org/10.1016/j.chemosphere.2009.02.033

[46] Sharma, I., Pati, P.K. and Bhardwaj, R. (2011) Effect of 28-Homobrassinolide on Antioxidant Defence System in Rapahnus sativus L. under Chromium Toxicity. Ecotoxicology, 20, 862-874. http://dx.doi.org/10.1007/s10646-011-0650-0

[47] Bajguz, A. (2011) Suppression of Chlorella vulgaris Growth by Cadmium, Lead and Copper Stress and Its Restoration by Endogenous Brassinolide. Archives of Environmental Contamination and Toxicology, 60, 406-416. http://dx.doi.org/10.1007/s00244-010-9551-0

[48] Ramakrishna, B. and Rao, S.S.R. (2013) Preliminary Studies on the Involvement of Glutathione Metabolism and Redox Status against Zinc Toxicity in Radish Seedlings by 28-Homobrassinolide. Environmental and Experimental Botany, 96, 52-58. http://dx.doi.org/10.1016/i.envexpbot.2013.08.003

\section{Submit or recommend next manuscript to SCIRP and we will provide best service for you:}

Accepting pre-submission inquiries through Email, Facebook, LinkedIn, Twitter, etc.

A wide selection of journals (inclusive of 9 subjects, more than 200 journals)

Providing 24-hour high-quality service

User-friendly online submission system

Fair and swift peer-review system

Efficient typesetting and proofreading procedure

Display of the result of downloads and visits, as well as the number of cited articles

Maximum dissemination of your research work

Submit your manuscript at: http://papersubmission.scirp.org/ 